\title{
On the readability of monotone Boolean formulae
}

\author{
Khaled Elbassioni · Kazuhisa Makino • Imran Rauf
}

Published online: 22 January 2010

(C) The Author(s) 2010. This article is published with open access at Springerlink.com

\begin{abstract}
Golumbic et al. (Discrete Appl. Math. 154:1465-1477, 2006) defined the readability of a monotone Boolean function $f$ to be the minimum integer $k$ such that there exists an $\wedge-\vee$-formula equivalent to $f$ in which each variable appears at most $k$ times. They asked whether there exists a polynomial-time algorithm, which given a monotone Boolean function $f$, in CNF or DNF form, checks whether $f$ is a read- $k$ function, for a fixed $k$. In this paper, we partially answer this question already for $k=2$ by showing that it is NP-hard to decide if a given monotone formula represents a read-twice function. It follows also from our reduction that it is NP-hard to approximate the readability of a given monotone Boolean function $f:\{0,1\}^{n} \rightarrow\{0,1\}$ within a factor of $\mathcal{O}(n)$. We also give tight sublinear upper bounds on the readability of a monotone Boolean function given in CNF (or DNF) form, parameterized by the number of terms in the CNF and the maximum size in each term, or more generally the maximum number of variables in the intersection of any constant number of terms. When the variables of the DNF can be ordered so that each term consists of a set of consecutive variables, we give much tighter logarithmic bounds on the readability.
\end{abstract}

Keywords Read-once functions · Monotone Boolean functions · Readability · Complexity of monotone Boolean functions

K. Elbassioni · I. Rauf ( $\varangle)$

Max-Planck-Institut für Informatik, Saarbrücken, Germany

e-mail: irauf@mpi-inf.mpg.de

K. Elbassioni

e-mail: elbassio@mpi-inf.mpg.de

K. Makino

Department of Mathematical Informatics, University of Tokyo, Tokyo, Japan

e-mail:makino@mist.i.u-tokyo.ac.jp 


\section{Introduction}

Let $f:\{0,1\}^{n} \rightarrow\{0,1\}$ be a monotone Boolean function, i.e., for any $x, x^{\prime} \in$ $\{0,1\}^{n}, x^{\prime} \geq x$ implies $f\left(x^{\prime}\right) \geq f(x)$. One property of such functions is that they can be represented by negation-free Boolean formulae. A minterm (maxterm) of monotone Boolean function $f\left(x_{1}, \ldots, x_{n}\right)$ is a minimal set of variables which, if assigned the value 1 (resp., value 0 ), forces the function to take the value 1 (resp., value 0 ) regardless of the values assigned to the remaining variables. It is well-known that the irredundant (i.e., no term contains another) disjunctive normal form (DNF) and conjunctive normal form (CNF) of monotone Boolean function $f$ consist respectively of all of its minterms and maxterms (cf. Wegener 1987).

A monotone read- $k$ formula is a Boolean formula over the operators $\{\vee, \wedge\}$ in which each variable occurs at most $k$ times. The readability of $f$ is the minimum $k$ such that $f$ can be represented by a monotone read- $k$ formula. We also call $f$ a read$k$ function when it has readability $k$. Finding the readability of an arbitrary Boolean function and computing a formula which achieves this readability has applications in circuit design among others and therefore is one of the earliest problems considered in Computer Science (Golumbic et al. 2006).

Given a monotone Boolean function in one of the normal forms (CNF/DNF), a complete combinatorial characterization for it to be read-once was given by Gurvich (1977, 1991). A polynomial-time algorithm based on this criterion is given by Golumbic et al. (2006) to decide whether a given CNF or DNF is read-once. The algorithm also computes the unique read-once representation when a read-once function is given as input. For $k \geq 2$, no characterization is known for a given monotone Boolean CNF or DNF to be read- $k$, and in fact, Golumbic et al. (2006) asked whether there exists a polynomial-time algorithm, which given a (normal) monotone Boolean function $f$ in CNF or DNF form, checks whether $f$ is a read- $k$ function, for a fixed $k$.

The case when the function is given by an oracle has also been considered in the machine learning community. It is shown in Angluin et al. (1993) that given a readonce function by a membership oracle, we can compute its read-once representation in polynomial time. However, the correctness of the algorithm is based on the assumption that the function provided as an oracle is read-once. If its not read-once then the algorithm terminates with incorrect output.

In this paper, we show that, given an $\wedge-\vee$-formula, it is NP-hard to check if it represents a read-twice function $f$. In fact, we prove a stronger result: given a readtwice representation of $f$, it is hard to decide whether $f$ is actually read-once. This partially answers the question of Golumbic et al. (2006), but leaves open the case when $f$ is given by the CNF or DNF normal form. It follows also from our reduction that it is NP-hard to approximate the readability of a given monotone Boolean function $f:\{0,1\}^{n} \rightarrow\{0,1\}$ within a factor of $\mathcal{O}(n)$.

It follows from a result in Wegener (1987) that almost all monotone Boolean functions on $n$ variables, in which each minterm has size exactly $k$, have readability $\Omega\left(n^{k-1} \log ^{-1} n\right)$. Assuming that the function is given by its irredundant DNF (or $\mathrm{CNF})$ of $m$ minterms, this implies a lower bound of $\tilde{\Omega}\left(m^{1-\frac{1}{k}}\right)$ on the readability. This naturally raises the question whether this bound is tight, i.e for any monotone 
CNF formula of $m$ terms, there exists an equivalent read- $O\left(m^{1-\frac{1}{k}}\right)$ representation. In this paper, we show that this indeed the case, and moreover that such a representation can be found in polynomial time. In fact, we prove a more general result. For integers $p, q>0$, let us say a monotone CNF $f$ has $(p, q)$-bounded intersection (Khachiyan et al. 2007) if every $p$ terms intersect in at most $q$ variables. We show that any such CNF has read- $O\left((p+q-1) m^{1-\frac{1}{q+1}}\right)$ representation which can be found in polynomial-time. Confronted with this almost tight sublinear bound on readability, an interesting question is whether it can be improved for interesting special cases. For the class of interval DNF's, i.e. those for which there is an ordering on the variables such that each term contains only consecutive variables in that ordering, we show that readability is at most $O(\log m)$.

The paper is organized as follows. In the next section, we point out that the characterization of Gurvich (1977) for read-once functions does not carry over to read- $k$ functions already for $k=2$. In Sect. 3, we present upper bounds on the readability of some classes monotone Boolean DNF (resp. CNF) that depends only on the number of terms in the normal form. In Sect. 4 we show that finding the readability in general is hard when the input formula is not a DNF or CNF. We also give an $\mathcal{O}(n)$ inapproximability result in this case.

\section{On generalization of read-once functions}

An elegant characterization of read-once functions is provided by the following theorem of Gurvich.

Theorem 1 (Gurvich 1977) For any monotone Boolean function $f$ the following two statements are equivalent: (i) $f$ is read-once. (ii) Every minterm and maxterm of $f$ intersect in exactly $c=1$ variable.

However, this result does not generalizes to read-twice functions as the following example shows. Consider the read-twice formula

$$
g\left(x_{1}, \ldots, x_{n}, y_{1}, \ldots, y_{n}\right)=\bigwedge_{1 \leq i \leq n}\left(x_{i} \vee y_{i}\right) \bigwedge\left(x_{1} \vee \ldots \vee x_{n}\right)
$$

It is easy to see that the $g$ has a minterm $x_{1} \ldots x_{n}$ which intersects with the maxterm $\left(x_{1} \vee \ldots \vee x_{n}\right)$ in $n$ variables. Hence hypergraphs corresponding to read-twice functions do not necessarily satisfy the generalization of Condition (ii) of Theorem 1 for any constant $c>1$. Conversely, any such generalization is also not sufficient for a function to be read- $c$, as implied by the following result on the shortest possible size of $k$-homogeneous DNF where the size of each term is exactly $k$ (and hence each minterm and maxterm intersect in at most $k$ ).

Theorem 2 (cf. Wegener 1987) For an integer $k$, let $\mathscr{H}_{k}^{n}$ be the class of monotone Boolean functions on $n$ variables such that size of every minterm is exactly $k$. The monotone formula size of almost all $h \in \mathscr{H}_{k}$ is $\Omega\left(n^{k} \log ^{-1} n\right)$. 
Theorem 2 implies that the readability of almost all $h \in \mathscr{H}_{k}$ is $\Omega\left(n^{k-1} \log ^{-1} n\right)$, since otherwise the formula achieving a smaller readability has smaller then shortest possible size.

\section{Upper bounds}

In this section, we consider various classes of monotone Boolean DNF's and give upper bounds on their readability. First we consider Interval DNF's whose terms correspond to consecutive variables, given some ordering on variables. Next, we consider $(p, q)$-intersecting DNF where every $p$ of its terms intersect in at most $q$ variables and give an almost tight upper bound on their readability. Finally, we consider a special case of the latter class, namely $k$-DNF, where the size of each term is bounded by $k$ and again give a tight upper bound on their readability. Even though we get the same upper bound implied by the more general case, the formula computed by our algorithm has only depth 3 in this case.

In our description of the algorithms, we use set-theoretic notations to describe various operations on the structure of DNF's. In this sense, we treat the DNF $\phi=\bigvee t_{i}$ as its corresponding hypergraph $\left\{t_{i} \mid t_{i}\right.$ is a term in $\left.\phi\right\}$. For example, we write $t \in \phi$ when $t$ is term of $\phi$ and similarly by $x \in t$ we mean that the term $t$ contains variable $x$. Let us denote the degree of a variable in $\phi$ by $\operatorname{deg}_{\phi}(x)$, which is the number of terms in $\phi$ containing $x \in V$. For a Boolean formula $f$ and a literal $x$ (resp. set of literals $S$ ) in $f$, we denote by $\left.f\right|_{x=1}$ (resp. $\left.f\right|_{S=1}$ ) the resulting $f$ after replacing every occurrence of $x$ (resp. $x \in S$ ) in $f$ with 1 .

\subsection{Interval DNF}

A monotone Boolean DNF $\mathcal{I}=\bigvee_{I \in \mathcal{I}} \bigwedge_{x \in I} x$ is called interval DNF if there is an ordering of variables $V=\left\{x_{1}, x_{2}, \ldots, x_{n}\right\}$ such that each $I \in \mathcal{I}$ contains only consecutive elements from the ordering. We show that an interval DNF containing $m$ terms is $\mathcal{O}(\log m)$-readable. For a term $I=x_{i} x_{i+1} \ldots x_{j}$ in interval DNF $\mathcal{I}$, we call $x_{i}$ and $x_{j}$ its left and right end-points, and denote them with $\mathrm{L}(I)$ and $\mathrm{R}(I)$ respectively. We also denote the first (resp. last) term in the ordering of terms of $\mathcal{I}$ with respect to their left end point as $\operatorname{first}(\mathcal{I})$ and last $(\mathcal{I})$ respectively.

Let us call an interval DNF intersecting if all terms in it have a non-empty intersection. It is known that the terms of an irredundant interval DNF can be partitioned into two classes such that each class is a union of disjoint intersecting DNF's (cf. Albertson et al. 1989). Consequently, the readability of an irredundant interval DNF can be bounded by twice the maximum readability of an intersecting DNF in the partition. The algorithm to find a $2\left\lceil\log \left(m^{\prime}\right)\right\rceil$-readable formula for intersecting DNF $\mathcal{I}^{\prime}$ consisting of $m^{\prime}$ terms is given in Fig. 1. It first divides the terms in $\mathcal{I}^{\prime}$ into two halves $\left(\mathcal{I}_{1}\right.$ and $\mathcal{I}_{2}$ ) by considering them in order with respect to their left end-point. The common variables are then factored out from $\mathcal{I}_{1}$ and $\mathcal{I}_{2}$ and the equivalent formulae for the remaining parts are computed recursively.

Theorem 3 Let $\mathcal{I}$ be an irredundant interval DNF containing $m$ terms. Then $\mathcal{I}$ is $4\lceil\log (m)\rceil$-readable. 


\section{Procedure REDUCE1 $(\mathcal{I})$ :}

Input: A monotone Boolean interval DNF $\mathcal{I}=\bigvee_{j=1}^{m} I_{j}$ s.t. $\bigcap_{j=1}^{m} I_{j} \neq \emptyset$

Output: $\mathrm{A} \mathcal{O}(\log m)$ readable formula $\psi$ equivalent to $\mathcal{I}$

1. if $|\mathcal{I}| \leq 2$ then return the read-once formula representing $\mathcal{I}$

2. Consider terms in $\mathcal{I}$ in order of their left end points, let $\mathcal{I}_{1}$ (resp. $\mathcal{I}_{2}$ ) be first half (resp. remaining half) elements of $\mathcal{I}$.

3. Let $\phi_{1}$ (resp. $\phi_{2}$ ) be maximum set of variables that occur in every term of $\mathcal{I}_{1}\left(\right.$ resp. $\left.\mathcal{I}_{2}\right)$

4. $t_{1}:=\operatorname{first}\left(\mathcal{I}_{1}\right), t_{2}:=\operatorname{last}\left(\mathcal{I}_{1}\right), t_{3}:=\operatorname{first}\left(\mathcal{I}_{2}\right), t_{4}:=\operatorname{last}\left(\mathcal{I}_{2}\right)$

5. $\psi_{1}=\operatorname{REDUCE} 2\left(\left.\left(\mathcal{I}_{1} \backslash\left\{t_{1}, t_{2}\right\}\right)\right|_{\phi_{1}=1}\right), \quad \psi_{2}=\operatorname{REDUCE} 2\left(\left.\left(\mathcal{I}_{2} \backslash\left\{t_{3}, t_{4}\right\}\right)\right|_{\phi_{2}=1}\right)$

6. return $\left(\phi_{1} \wedge\left(\left.\left.\psi_{1} \vee t_{1}\right|_{\phi_{1}=1} \vee t_{2}\right|_{\phi_{1}=1}\right)\right) \bigvee\left(\phi_{2} \wedge\left(\left.\left.\psi_{2} \vee t_{3}\right|_{\phi_{2}=1} \vee t_{4}\right|_{\phi_{2}=1}\right)\right)$

Fig. 1 An algorithm to find an $2\lceil\log (m)\rceil$-readable formula for interval DNF consisting of $m$ intersecting terms

Proof We first partition the terms of $\mathcal{I}$ into two classes such that each class is the union of disjoint intersecting DNF. For each intersecting DNF $\mathcal{I}^{\prime}$ in either of the classes, let $r\left(m^{\prime}\right)$ be the readability of the formulae generated by the procedure REDUCE1 $\left(\mathcal{I}^{\prime}\right)$ when given an interval DNF $\mathcal{I}^{\prime}$ containing $m^{\prime}$ intersecting terms as input. We show that $r\left(m^{\prime}\right)$ is $2\left\lceil\log \left(m^{\prime}\right)\right\rceil$ which implies that the readability of $\mathcal{I}$ is $4\lceil\log (m)\rceil$.

Given an intersecting interval DNF $\mathcal{I}^{\prime}$ the procedure REDUCE1 $\left(\mathcal{I}^{\prime}\right)$ divides the problem into subproblems $\mathcal{I}_{1}$ and $\mathcal{I}_{2}$ respectively. Note that the subproblems in the recursive call i.e. $\left(\left.\mathcal{I}_{1} \backslash\left\{\operatorname{first}\left(\mathcal{I}_{1}\right), \operatorname{last}\left(\mathcal{I}_{1}\right\}\right)\right|_{\phi_{1}=1}\right.$ and $\left.\left(\mathcal{I}_{2} \backslash\left\{\operatorname{first}\left(\mathcal{I}_{2}\right), \operatorname{last}\left(\mathcal{I}_{2}\right)\right\}\right)\right|_{\phi_{2}=1}$ are again intersecting since $\mathcal{I}_{1}$ and $\mathcal{I}_{2}$ are irredundant. For calculating the readability of the formula computed by $\operatorname{REDUCE} 1\left(\mathcal{I}^{\prime}\right)$, consider the case when a variable $x_{i}$ occurs in both subproblems. We show that if $x_{i}$ does not occur in $\phi_{1}$ (resp. $\phi_{2}$ ) then it is necessarily the case that it appears in $\phi_{2}$ (resp. $\phi_{1}$ ) and thus occurring only once in at least one of the subproblems. Note that since $\mathcal{I}^{\prime}$ is irredundant, the set $\phi_{2}$ forms the interval $\left[\mathrm{L}\left(\operatorname{last}\left(\mathcal{I}_{2}\right)\right), \mathrm{R}\left(\operatorname{first}\left(\mathcal{I}_{2}\right)\right)\right]$. Also observe that since $x_{i}$ occurs in both subproblems and not in $\phi_{1}$, it must lie in the interval [R(first $\left.\left.\left(\mathcal{I}_{1}\right)\right), \operatorname{R}\left(\operatorname{last}\left(\mathcal{I}_{1}\right)\right)\right]$. It is easy to see that the later interval is the subset of $\phi_{2}$ since $\mathrm{R}\left(\operatorname{last}\left(\mathcal{I}_{1}\right)\right)$ appears before $\mathrm{R}\left(\right.$ first $\left(\mathcal{I}_{2}\right)$ ) in the ordering of variables because of the definition of $\mathcal{I}_{1}$ and $\mathcal{I}_{2}$. Also because of the assumption that $\mathcal{I}^{\prime}$ is intersecting, $\operatorname{L}\left(\operatorname{last}\left(\mathcal{I}_{2}\right)\right)$ appears before $\mathrm{R}\left(\right.$ first $\left.\left(\mathcal{I}_{1}\right)\right)$ in the ordering. So the maximum readability of the formula generated by REDUCE1 $\left(\mathcal{I}^{\prime}\right)$ where $\mathcal{I}^{\prime}$ consists of $m^{\prime}$ terms satisfies $r\left(m^{\prime}\right) \leq 2+r\left(\left\lceil m^{\prime} / 2\right\rceil\right)$. Solving the recurrences yields the stated bound on the readability of $\mathcal{I}^{\prime}$.

\section{$3.2(p, q)$-intersecting DNF}

A monotone Boolean DNF is called $(p, q)$-intersecting if every $p$ of its distinct terms intersect in at most $q$ variables. A quadratic DNF for instance is $(2,1)$-intersecting and $k$-DNF, i.e., DNF where the size of each term is bounded by $k$ is $(2, k-1)$ intersecting. In this section, we give a $(p+q-1) m^{1-\frac{1}{q+1}}$ bound on the readability of $(p, q)$-intersecting DNF containing $m$-terms. Theorem 2 implies that this bound is almost tight because by considering $q+1$-homogeneous DNF containing $m=$ $\Theta\left(n^{q+1}\right)$ terms we get, 


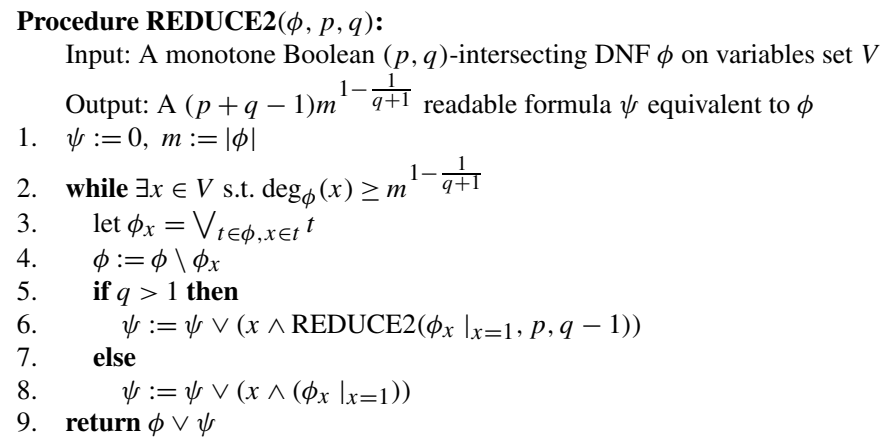

Fig. 2 An algorithm to find $(p+q-1) m^{1-\frac{1}{q+1}}$ readable formula for $(p, q)$-intersecting DNF consists of $m$ terms

Corollary 1 For a constant $q$, let $q_{q}$ be the class of monotone Boolean DNF on $n$ variables with $m$ terms such that size of every minterm is exactly $q+1$. The readability of almost all $g \in g_{q}$ is $\Omega\left(m^{1-\frac{1}{q+1}} \log ^{-1} n\right)$.

Let $\phi$ be a $(p, q)$-intersecting monotone Boolean DNF on variables $V=$ $\left\{x_{1}, \ldots, x_{n}\right\}$. The algorithm is given in Fig. 2. It works by picking a variable $x$ with high degree in $\phi$ and recursively computing a formula equivalent to the part of $\phi$ where $x$ occurs. The algorithm stops when every variable has low degree in the remaining expression. More precisely, for a variable $x \in V$, let $\phi_{x}$ be the DNF consisting of terms of $\phi$ which contain $x$, i.e. $\phi_{x}=\bigvee_{t \in \phi, x \in t} t$. Note that if $\phi$ is $(p, q)$-intersecting then $\left.\phi_{x}\right|_{x=1}$ is $(p, q-1)$-intersecting DNF, so the algorithm recurs when $q>1$ and otherwise it returns the read- $(p-1)$ formula $x \wedge\left(\left.\phi_{x}\right|_{x=1}\right)$. The next Theorem bounds the readability of the formula generated by the algorithm.

Theorem 4 Given a monotone Boolean DNF $\mu$ which is $(p, q)$-intersecting for $p \geq 2, q \geq 1$. The formula $\mu^{\prime}=\operatorname{REDUCE} 2(\mu, p, q)$ is $(p+q-1) m^{1-\frac{1}{q+1}}$ readable and it is equivalent to $\mu$.

Proof The proof is by induction on $q$. When $q=1$, the while loop in Step 2 ensure that every variable in $\phi$ has degree less then $\sqrt{m}$ after the loop ends. Moreover, a read$(p-1)$ formula is added to $\psi$ in each iteration of while loop. Since there are at most $\sqrt{m}$ iterations, the formula $\phi \vee \psi$ in Step 9 has readability at most $\sqrt{m}+(p-1) \sqrt{m}$.

Now assume that the claim is true for $(p, q-1)$ intersecting DNF, where $q \geq 2$. We prove it for $(p, q)$-intersecting DNF using similar arguments as in the previous paragraph. After the while loop ends, every variable in the remaining $\phi$ has degree less then $m^{1-\frac{1}{q+1}}$. Let $m_{1}, \ldots, m_{d}$ be number of terms removed from $\phi$ in each iteration of while loop, where $d$ is the number of iterations. Note that $d$ can be bounded from above by $m^{\frac{1}{q+1}}$ since each $m_{i}$ is at least $m^{1-\frac{1}{q+1}}$. Now, denoting the readability 
of $(p, q)$-intersecting DNF on $m$ terms by $r_{p, q}(m)$, we have

$$
\begin{aligned}
r_{p, q}(m) & \leq m^{1-\frac{1}{q+1}}+\sum_{i=1}^{d} r_{p, q-1}\left(m_{i}\right) \leq m^{1-\frac{1}{q+1}}+\sum_{i=1}^{d}\left((p+q-2) m_{i}^{1-\frac{1}{q}}\right) \\
& \leq m^{1-\frac{1}{q+1}}+(p+q-2) d\left(\frac{\sum_{i=1}^{d} m_{i}}{d}\right)^{1-\frac{1}{q}} \leq(p+q-1) m^{1-\frac{1}{q+1}},
\end{aligned}
$$

where we apply induction hypothesis to get (1) and use Jensen's inequality to get (2).

The correctness of the procedure is straightforward since the invariant that $\phi \vee \psi$ is equal to $\mu$ holds after completion of every iteration.

Note that the algorithm produces a depth $q$ formula. In the next section we will see that we can do much better in this regard for the a subclass of $(p, q)$-intersecting DNF, namely the class of DNF where the size of each term is bounded by a constant $k$.

\section{$3.3 k-\mathrm{DNF}$}

A monotone Boolean DNF is called $k$-DNF if every term in it has size at most $k$. In this section, we give an algorithm to compute $2 \mathrm{~km}^{1-1 / k}$ readable formula of depth three and equivalent to given $k$-DNF. We need the following definitions.

A sunflower with p petals and a core $Y$ is a collection of sets $S_{1}, \ldots, S_{p}$ such that $S_{i} \cap S_{j}=Y$ for all $i \neq j$ and none of the sets $S_{i}-Y$ is empty. We allow the core $Y$ to be empty however, so every pairwise disjoint family of sets constitutes a sunflower.

Lemma 1 (Sunflower Lemma (Erdös and Rado 1960)) Let $\mathcal{H} \subseteq 2^{V}$ be a hypergraph with $m=|\mathcal{H}|$ and size of each edge is bounded by $k$. If $m>k ! p^{k}$ then $\mathcal{H}$ contains a sunflower with $p+1$ petals.

Since a sunflower has a straightforward read-once representation, the above lemma immediately gives an upper bound on the readability of $k$-DNF with $m$ terms. The algorithm works by finding a sunflower with certain minimal size, representing them as read-once formula and recurse on the remaining edges.

Theorem 5 Let $f$ be a monotone Boolean DNF with $m$ terms such that the size of each term in $f$ is bounded by $k$ then $f$ is $2 \mathrm{~km}^{1-1 / k}$-readable. Moreover, a formula of such readability and depth 3 can be found in polynomial time.

Proof Any $k$-DNF with $m$ terms contains a sunflower of size at least $(m / k !)^{1 / k}$ which we remove and recurse on the remaining terms. Let $r(m)$ denote the readability of boolean $k$-DNF with $m$ terms then the readability of $f$ can be bounded by the recurrence $r(m) \leq 1+r\left(m-(m / k !)^{1 / k}\right)$ with $r(2)=r(1)=1$. By using the inequality $k ! \leq k^{k}$ and substituting $r(m)=2 \mathrm{~km}^{1-1 / k}$ in the above recurrence we get $g(k, m)=2 k m^{1-\frac{1}{k}}\left(1-\left(1-\frac{m^{\frac{1}{k}-1}}{k}\right)^{1-\frac{1}{k}}\right) \geq 1$. In Appendix we prove the following claim: 
Claim 1 For $k \geq 2$ and $m \geq 1$, the function $g(k, m)$ is monotonically decreasing in $m$ and monotonically increasing in $k$.

Thus the minimum of $g$ is attained when $k=2$ and $m$ approaches infinity. The minimum value is 1 and hence $r(m) \leq 2 \mathrm{~km}^{1-1 / k}$. Finally, we note that the proof of Lemma 1 is constructive and a sunflower of desired size can be computed in time polynomial in number of variables and terms of a DNF.

\section{Hardness and inapproximability}

In this section, we show that finding the readability of a given monotone Boolean formula is NP-hard. The reduction we use is gap-introducing and so it also gives hardness of approximating readability unless $P=N P$. Our reduction is from the well-known NP-complete problem of deciding satisfiability of a given Boolean 3CNF $\Phi\left(x_{1} \ldots x_{n}\right)=\bigwedge_{j=1}^{m} \Phi_{j}$. For all $i \in[n]$ and $j \in[m]$, let us define new variables $y_{i j}, y_{i j}^{\prime}, z_{i j}^{\prime}$ for a literal $x_{i}$ in clause $\Phi_{j}$ and variables $z_{i j}, z_{i j}^{\prime}, y_{i j}^{\prime}$ for a literal $\neg x_{i}$ in clause $\Phi_{j}$. Let $\phi\left(y_{11} \ldots y_{n m}, z_{11} \ldots z_{n m}\right)$ be the monotone CNF we get from $\Phi\left(x_{1} \ldots x_{n}\right)$ by substituting $y_{i j}$ for $x_{i}$ in $\Phi_{j}$ and $z_{i^{\prime} j}$ for $\neg x_{i^{\prime}}$ in $\Phi_{j}$ such that $\phi(y, z) \equiv \Phi(x)$, for $y_{i j}=x_{i}$ and $z_{i j}=\neg x_{i}, i \in[n], j \in[m]$. Furthermore, let $I_{i}=\left\{j: x_{i} \in \Phi_{j}\right\} \cup\left\{j: \neg x_{i} \in \Phi_{j}\right\}$, we define

$$
\rho\left(y^{\prime}, z^{\prime}\right)=\bigwedge_{i=1}^{n}\left(\bigwedge_{j \in I_{i}} y_{i j}^{\prime} \vee \bigwedge_{j \in I_{i}} z_{i j}^{\prime}\right), \quad \psi\left(y, z, y^{\prime}, z^{\prime}\right)=\bigvee_{x_{i} \in \Phi_{j}} y_{i j} z_{i j}^{\prime} \vee \bigvee_{\neg x_{i} \in \Phi_{j}} y_{i j}^{\prime} z_{i j} .
$$

Now consider the following Boolean function

$$
f\left(y, z, y^{\prime}, z^{\prime}\right)=\left(\phi(y, z) \bigwedge \rho\left(y^{\prime}, z^{\prime}\right)\right) \bigvee \psi\left(y, z, y^{\prime}, z^{\prime}\right) .
$$

Note that the size of $f$ is $15 m$, where $m$ is number of clauses in $\Phi$. The next lemma shows that finding the readability of Boolean formula $f$ defined in (3) is equivalent to solving satisfiability for $\Phi(x)$.

Lemma 2 The monotone Boolean function $f$ in (3) is read-2 if and only if $\Phi(x)$ is satisfiable. It is read-once otherwise.

Proof Denote the two disjuncts in $f$ by $f_{1}=\phi(y, z) \wedge \rho\left(y^{\prime}, z^{\prime}\right)$ and $f_{2}=\psi(y, z$, $\left.y^{\prime}, z^{\prime}\right)$. We first show that the minterms of $f_{1}$ which are not absorbed by minterms of $f_{2}$ correspond precisely to the satisfiable assignments of $\Phi$ and so $f=\psi$ is clearly a read-once function if $\Phi$ is not satisfiable.

Let $\hat{x}$ be a satisfiable assignment of $\Phi(x)$. Since $\hat{x}$ makes at least one literal true in each clause of $\Phi(x)$, the set $t_{\phi}=\left\{y_{i j} \mid \hat{x}_{i}=1\right\} \cup\left\{z_{i j} \mid \hat{x}_{i}=0\right\}$ contains a minterm $t_{\phi}^{\prime}$ of $\phi(y, z)$. Similarly, note that the set $t_{\rho}=\left\{y_{i j}^{\prime} \mid \hat{x}_{i}=1\right\} \cup\left\{z_{i j}^{\prime} \mid \hat{x}_{i}=0\right\}$ defines a minterm of $\rho\left(y^{\prime}, z^{\prime}\right)$, and so the set $t=t_{\phi}^{\prime} \cup t_{\rho}$ is a minterm of $f_{1}$. It is easy to check that $t$ does not contain any minterm of $f_{2}$ since for all $i \in[n]$ and $j \in[m]$, at most one from each pair $y_{i j}, z_{i j}^{\prime}$ and $y_{i j}^{\prime}, z_{i j}$ are members of $t$. 


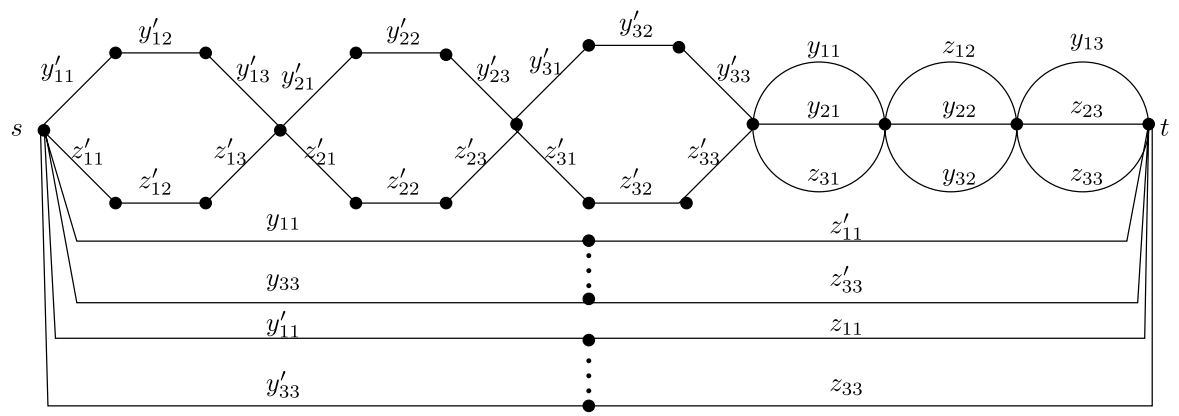

Fig. 3 Applying reduction in (3) to 3-CNF $\Phi=\left(x_{1} \vee x_{2} \vee \neg x_{3}\right)\left(\neg x_{1} \vee x_{2} \vee x_{3}\right)\left(x_{1} \vee \neg x_{2} \vee\right.$ $\neg x_{3}$ ). Minimal $s-t$ paths in the figure correspond to minterms of $f$, whereas minimal $s-t$ cuts are maxterms of $f$

Conversely, any minterm $t$ of $f_{1}$ contains one of $y_{i 1}^{\prime} \ldots y_{i m}^{\prime}$ or $z_{i 1}^{\prime} \ldots z_{i m}^{\prime}$ for all $i \in[n]$ to cover the conjunct $\rho$. Assume $t$ is not absorbed by any term of $f_{2}$. Consequently, $t$ does not contain both $y_{i j} z_{i j}^{\prime}$ or $y_{i j}^{\prime} z_{i j}$ for all $i \in[n]$ and $j \in[m]$. Therefore it must contain from each clause $\phi_{j}$, at least one of the variable $y_{i j}$ or $z_{i j}$ consistent with the primed variable selected from $\rho$. Hence the assignment $x_{i}=1$ if $y_{i j} \in t$ and $x_{i}=0$ if $z_{i j} \in t$ satisfies $\Phi(x)$.

It only remains to prove that $f$ is not a read-once function when $\Phi(x)$ is satisfiable. Assume without loss of generality that the variable $x_{1}$ appears in clause $\Phi_{1}$. Let us define a maxterm $c$ of $f$ by $c=\left\{y_{11}^{\prime}, z_{11}^{\prime}\right\} \bigcup_{i \in[n], j \in[m]}\left\{y_{i j}, y_{i j}^{\prime}\right\}$ and consider the minterm $t$ of $f$ corresponding to a satisfiable assignment $\hat{x}$ of $\Phi$ as defined above. It is easy to see that $|t \cap c|>1$ since for any literal $x_{i}$ appears in clause $\Phi_{j}$ such that $\hat{x}_{i}=1, t$ would contain both $y_{i j}$ and $y_{i j}^{\prime}$. Hence $f$ is not a read-once function because of Theorem 1. Note that it is read-2 since we have (3) as its read-2 representation.

Since $f$ in (3) is compose of two read-once formulae, Lemma 2 also implies the hardness of determining if a given monotone formula is disjunction of two read-once formulae.

Corollary 2 It is NP-hard to decide whether a given disjunction of two monotone read-once functions is a read-once function.

A weaker form of Corollary 2 can be deduced from the constructions in Gurvich and Khachiyan (1999) and Boros et al. (2008), which can be used to show that NPhardness of deciding if a given read-3 monotone formula is actually read-once.

Another interesting problem for which we get a hardness result as a corollary of Lemma 2 is the problem of generating all minterms or maxterms of given monotone Boolean formula. Note that the problem can be solved in polynomial time (Golumbic and Gurvich 2010) when the input formula is read-once.

Lemma 3 Let $\mathcal{F}$ be the class of monotone Boolean formulae in which each variable appears at most twice. For a formula $f \in \mathcal{F}$, let $C$ and $D$ denote the sets of the maxterms and the minterms of $f$, respectively. 
(i) Given a formula $f \in \mathcal{F}$ and a subset $C^{\prime}$ of $C$, it is coNP-complete to decide whether $C^{\prime}=C$.

(ii) Similarly, for a formula $f \in \mathcal{F}$ and a subset $D^{\prime}$ of $D$, it is coNP-complete to decide whether $D^{\prime}=D$.

Proof Note that since the class $\mathcal{F}$ is closed under duality, both parts of the theorem are equivalent. The hardness of (ii) implied immediately from Lemma 2 by setting $D^{\prime}=\{t \mid t$ is a term in $\psi\}$. The (possibly) remaining minterms in $D \backslash D^{\prime}$ correspond to satisfiable assignments of $\Phi$.

In the following, we generalize the reduction introduced in (3) and get an inapproximability result for the problem of determining readability of given monotone Boolean formula. We use a result Gál (2002) that gives an explicit monotone Boolean function $\alpha$ on $s$ variables such that the size of the shortest monotone formula representing $\alpha$ is $s^{\Omega(\log s)}$, moreover its irredundant monotone DNF has size $s^{\mathcal{O}(\log s)}$. Note that the readability of $\alpha$ is also $s^{\Omega(\log s)}$, since otherwise we could represent $\alpha$ by a formula with smaller then shortest possible size. We define the following reduction

$$
f^{\prime}\left(w, y, z, y^{\prime}, z^{\prime}\right)=\left(\phi(y, z) \bigwedge \rho\left(y^{\prime}, z^{\prime}\right) \bigwedge \alpha(w)\right) \bigvee \psi\left(y, z, y^{\prime}, z^{\prime}\right),
$$

where the size of $f^{\prime}$ is $15 m+s^{\mathcal{O}(\log s)}$. Note that if $\Phi$ is satisfiable, $f^{\prime}$ has readability $s^{\Theta(\log s)}$ by applying the same reasoning as in Lemma 2. By choosing $s$ and $m$ such that $m=s^{c_{1} \log s}$ and $m=c_{2} n$ for a suitable constants $c_{1}, c_{2}$, we get the following.

Corollary 3 There is no polynomial-time algorithm to approximate the readability of a given monotone Boolean formula $f$ within factor of $\mathcal{O}(n)$, unless $\mathrm{P}=\mathrm{NP}$.

Open Access This article is distributed under the terms of the Creative Commons Attribution Noncommercial License which permits any noncommercial use, distribution, and reproduction in any medium, provided the original author(s) and source are credited.

\section{Appendix}

Proof of the Claim 1 Assume for the rest of the proof that $k \geq 2$ and $m \geq 1$. We first observe that the derivative of $g$ with respect to $k$ is positive and so $g$ is increasing in $k$. Substituting $x=m^{-1+\frac{1}{k}} / k$, we obtain,

$$
\begin{aligned}
\frac{\partial g}{\partial k}= & 2\left(1-(1-x)^{1-\frac{1}{k}}\right) m^{1-\frac{1}{k}}+\frac{2\left(1-(1-x)^{1-\frac{1}{k}}\right) \log (m) m^{1-\frac{1}{k}}}{k} \\
& -2 k(1-x)^{1-\frac{1}{k}}\left(\frac{\left(1-\frac{1}{k}\right)\left(\frac{x}{k}+\frac{x \log m}{k^{2}}\right)}{1-x}+\frac{\log (1-x)}{k^{2}}\right) m^{1-\frac{1}{k}} \\
= & 2 m^{1-\frac{1}{k}}\left(\left(1-(1-x)^{1-\frac{1}{k}}\right)\left(1+\frac{\log m}{k}\right)\right.
\end{aligned}
$$




$$
\begin{aligned}
& \left.-k(1-x)^{1-\frac{1}{k}}\left(\frac{x\left(1-\frac{1}{k}\right)\left(1+\frac{\log m}{k}\right)}{k(1-x)}+\frac{\log (1-x)}{k^{2}}\right)\right) \\
= & 2 m^{1-\frac{1}{k}}\left(1+\frac{\log m}{k}\right)\left(\left(1-(1-x)^{1-\frac{1}{k}}\right)\right. \\
& \left.-\left(\frac{x\left(1-\frac{1}{k}\right)}{(1-x)^{\frac{1}{k}}}+\frac{\log (1-x)(1-x)^{1-\frac{1}{k}}}{k\left(1+\frac{\log m}{k}\right)}\right)\right) \\
= & 2 m^{1-\frac{1}{k}}\left(1+\frac{\log m}{k}\right)\left(1-\frac{1-2 x+\frac{x}{k}}{(1-x)^{\frac{1}{k}}}-\frac{\log (1-x)(1-x)^{1-\frac{1}{k}}}{k\left(1+\frac{\log m}{k}\right)}\right)
\end{aligned}
$$

Only the last factor in the above equation has negative terms. Note that $x=m^{-1+\frac{1}{k}} / k$ is always positive and at most $1 / 2$ when $k \geq 2$ and $m \geq 1$. Consequently, the term $\left(1-2 x+\frac{x}{k}\right) /(1-x)^{\frac{1}{k}}$ has value at most 1 and so results in positive quantity when subtracted from 1 . Furthermore, the factor $\log (1-x)$ is negative and therefore makes the last term positive as well.

Similarly, the derivative of $g$ with respect to $m$ is,

$$
\begin{aligned}
\frac{\partial g}{\partial m} & =2\left(1-\frac{1}{k}\right)\left(k\left(1-\left(1-\frac{m^{\frac{1}{k}-1}}{k}\right)^{1-\frac{1}{k}}\right) m^{-1 / k}+\frac{\left(\frac{1}{k}-1\right)\left(1-\frac{m^{\frac{1}{k}-1}}{k}\right)^{-1 / k}}{m}\right) \\
& =\frac{2\left(1-\frac{1}{k}\right)}{m\left(1-\frac{m^{\frac{1}{k}-1}}{k}\right)^{\frac{1}{k}}}\left(k m^{1-\frac{1}{k}}\left(\left(1-\frac{m^{\frac{1}{k}-1}}{k}\right)^{\frac{1}{k}}+\left(1-\frac{m^{\frac{1}{k}-1}}{k}\right)\right)+\frac{1}{k}-1\right)
\end{aligned}
$$

which is negative since the term $k m^{1-\frac{1}{k}}\left(\left(1-\frac{m^{\frac{1}{k}-1}}{k}\right)^{\frac{1}{k}}+\left(1-\frac{m^{\frac{1}{k}-1}}{k}\right)\right)$ is increasing and approaches $\frac{1}{k}-1$ as $m$ goes to infinity.

\section{References}

Albertson M, Jamison R, Hedetniemi S, Locke S (1989) The subchromatic number of a graph. Discrete Math 74(1-2):33-49

Angluin D, Hellerstein L, Karpinski M (1993) Learning read-once formulas with queries. J Assoc Comput Math 40(1):185-210

Boros E, Elbassioni K, Gurvich V, Makino K (2008) Generating vertices of polyhedra and related monotone generation problems. In: Avis D, Bremner D, Deza A (eds) CRM Proceedings \& Lecture Notes, Centre de Recherches Mathématiques at the Université de Montréal, vol 48. Am Math Soc, Providence, pp 15-39. Special issue on Polyhedral Computation

Erdös P, Rado R (1960) Intersection theorems for systems of sets. J London Math Soc 35:85-90

Gál A (2002) A characterization of span program size and improved lower bounds for monotone span programs. Comput Complex 10(4):277-296

Golumbic MC, Gurvich V (2010) Read-once functions. In: Crama Y, Hammer PL (eds) Boolean functions: theory, algorithms and applications. Cambridge University Press, Cambridge (in press)

Golumbic MC, Mintz A, Rotics U (2006) Factoring and recognition of read-once functions using cographs and normality and the readability of functions associated with partial k-trees. Discrete Appl Math 154(10):1465-1477 
Gurvich V (1977) On repetition-free boolean functions. Usp Mat Nauk 32:183-184 (in Russian)

Gurvich V (1991) Criteria for repetition-freeness of functions in the algebra of logic. Sov Math Dokl 43(3):721-726

Gurvich V, Khachiyan L (1999) On generating the irredundant conjunctive and disjunctive normal forms of monotone Boolean functions. Discrete Appl Math 96-97(1):363-373 http://dx.doi.org/ 10.1016/S0166-218X(99)00099-2

Khachiyan L, Boros E, Elbassioni KM, Gurvich V (2007) On the dualization of hypergraphs with bounded edge-intersections and other related classes of hypergraphs. Theor Comput Sci 382(2):139-150

Wegener I (1987) The complexity of Boolean functions. Wiley, New York 